\title{
A new collaborative-shared control strategy for continuous elder/robot assisted navigation
}

\author{
C. Urdiales ${ }^{1}$, J. M. Peula ${ }^{1}$, C. Barrué ${ }^{2}$, E. J. Pérez ${ }^{1}$, I.Sánchez-Tato ${ }^{1}$, J.C. del Toro ${ }^{1}$, U. \\ Cortés $^{2}$, F. Sandoval ${ }^{1}$, R. Annicchiarico ${ }^{3} \&$ C. Caltagirone ${ }^{3}$ \\ ${ }^{I}$ Dpto. Tecnología Electrónica, University of Malaga, Malaga, Spain \\ ${ }^{2}$ Software Department, Technical University of Catalonia, Barcelona, Spain \\ ${ }^{3}$ Fondazione Santa Lucia, Rome, Italia
}

\begin{abstract}
In nowadays aging society, many people require mobility assistance. Autonomous wheelchairs may provide some help, but they are not supposed to overtake all control on human mobility, as this is reported to lead to loss of residual capabilities and frustration. Instead, persons and wheelchairs are expected to cooperate. Traditionally, shared control hands control from human to robot depending on a triggering event. In this paper, though, we propose a method to allow constant cooperation between humans and robots, so that both have some weight in the emergent navigating behavior. We have tested the proposed method on a robotized Meyra wheelchair at Santa Lucia Hospedale in Rome with several volunteering in-patients presenting different disabilities. Results in indoor environments have been satisfactory both from a quantitative and qualitative point of view.
\end{abstract}

\section{INTRODUCTION}

A utonomy in an agent can be defined as the ability to perform activities independently. It has been reported that mobility is of key importance for a person to be autonomous. However, a physically and/or cognitive challenged person may require some assistance to achieve autonomous navigation, either from a machine or from other persons. Lack of human resources to assist elder people leads naturally to create systems to do it in an autonomous way.

Specifically, the most typical mobility assistive devices are power wheelchairs. However, not every person can have full control over a power wheelchair. Poor use of technology can result in systems that are difficult to learn or use and even may lead to catastrophic errors. Robotics has traditionally offered an alternative to human controlled wheelchairs. A mobile is considered to be autonomous when it can perform a task in a dynamic environment without continuous human guidance. It can be observed that most approaches to shared control usually rely on swapping control from human to machine according to more or less complex algorithms.

In this paper, we propose a new-shared control approach. Its main novelty is that the machine and the human, specially elders, cooperate all the time to achieve a better combined result in situations where

Manuscript revised on May 12, 2008. one or the other perform better, but also to keep the human always in charge of his/her own navigation. Our approach relies on locally evaluating the performance of the human and the wheelchair for each given situation. Then, both their motion commands are weighted according to those efficiencies and combined in a reactive way. This approach benefits from the advantages of typical reactive behaviours to combine different sources of information in a simple, seamless way into an emergent trajectory. We have tested the system with 30 volunteers presenting different disabilities ranging from minor to mild and a Meyra wheelchair modified to run an autonomous navigation system. All tests have been performed in the FSL in Rome under engineers and doctors supervision. Performances were carefully evaluated using common metrics in wheelchair navigation and also new ones proposed specifically for this type of control. We have also developed a representation strategy to visually evaluate efficiency at each point of a trajectory in terms of the local efficiency factors.

\section{COLLABORATIVE NAVIGATION}

\section{A. Wheelchair control}

Situations where machines and persons cooperate to achieve a common goal fall within the field of collaborative control. Depending on how much autonomy the machine has, collaborative approaches for human/machine control can be roughly categorized into: i) safeguarded operation; and ii) shared control. In the first case mobiles can be totally controlled by humans, but in some cases the vehicle makes some decisions to avoid imminent danger or when human control is not adequate.

Wheelchair navigation is particularly concerned with shared control, where control may be handled from user to machine depending on the situation at hand. A first group of approaches leaves control mostly to the person, and automatic navigation is only triggered when a given situation is detected, like 
imminent collision. Under these circumstances, a reactive algorithm [1][2][3] is used to avoid obstacles.

A second group of approaches [4][5][6] rely on using a basic set of primitives like AvoidObstacle, FollowWall or PassDoorway to assist the person in difficult maneuvers, either by manual selection or automatic triggering. The operator may guide the robot directly, or switch among various autonomous behaviors to deal with complex situations.

Also, the human operator might only point the target and the machine would be in charge of motion planning and path tracking on its own [7][8][9][10] These systems work like a conventional autonomous robot: the user simply provides a destination and the mobile is totally in charge of getting there via a hybrid navigation scheme. At any point, the user may override automatic control and take over.

In this paper, we choose to develop a new shared control paradigm where human and wheelchair cooperate all through the trajectory, without switching control from one to the other in an abrupt way. Person and wheelchair commands are linearly combined depending on the efficiency at each given time instant, so that the wheelchair just helps the person when he/she needs it. This approach has been chosen to avoid loss of residual capabilities due to an excess of help.

\section{B. Cooperative navigation}

In order to collaborate through a trajectory, humans may provide motion commands through any human/computer interface (HCI), typically a joystick, depending on their condition. Pads, voice and specific devices for people with severe mobility problems have also been used in literature.

If persons can not control the wheelchair on their own, wheelchairs may provide their own motion commands as well, using their own input sensors to evaluate the environment and planning algorithms to make decisions. In most cases, these sensors return range measurement, indicating how far the system is from nearby obstacles.

Specifically, in this work wheelchair commands are calculated via a pure Potential Fields Approach (PFA)[11]. Reactive navigation schemes are particularly interesting because they may manage several sensors and goals in a simple way, so they can be used to combine the human and wheelchair commands and goals. Thus, we use the joystick as a new goal to the reactive algorithm and combine it with the original PFA output, weighting each command vector with their own efficiency and a couple of constants (K1 and K2) to contemplate external (caregivers) opinions (Fig. 1). K1 and K2 are fixed in this work to 0.5 both, so that control is equally shared by human and robot, as we have still not included diagnosis nor biometrics in shared control. The shared output trajectory at position $p$ is obtained as:

$$
U(p)=K_{h} w_{h} J o y s(p)+K_{r} w_{r} P F A(p)
$$

The weights of the joystick and the robot $\left(\mathrm{w}_{\mathrm{h}}\right.$ and $\mathrm{w}_{\mathrm{r}}$ ) are a function of their local efficiencies in terms of smoothness, safety and directiveness, estimated as:

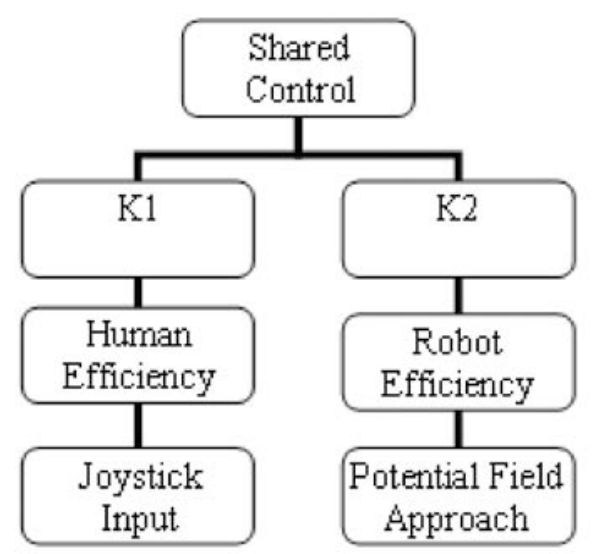

Fig. 1. System architecture

$$
E=\frac{k_{1} e^{-C_{1} \cdot \frac{1}{d_{\min }}}+k_{2} e^{-C_{2}\left|\alpha_{1}\right|}+k_{3} e^{-C_{3}\left|\alpha_{2}\right|}}{k_{1}+k_{2}+k_{3}}
$$

wimlinallu allu tile juysuln vutput iv votall $\mathrm{w}_{\mathrm{h}}$ allu $\mathrm{W}_{\mathrm{r}}$, respectively. All involved factors are presented in Fig.2.

$k_{i}$ control how important each of the factors is for global efficiency. $C_{i}$ weights the slope of the importance of each factor with respect to the baseline value. $d_{\min }$ is the minimum distance to obstacle detected by onboard range sensors with respect to the proposed output heading. $\alpha_{\text {goal }}$ is the angle difference between the output heading and the direction to the goal, representing that it is better to reach the goal following a straight line in terms of trajectory length. $\alpha_{\text {head }}$ is the angle difference between the output and input heading, representing that sharp turns should be avoided for safety and to minimize slippage. 


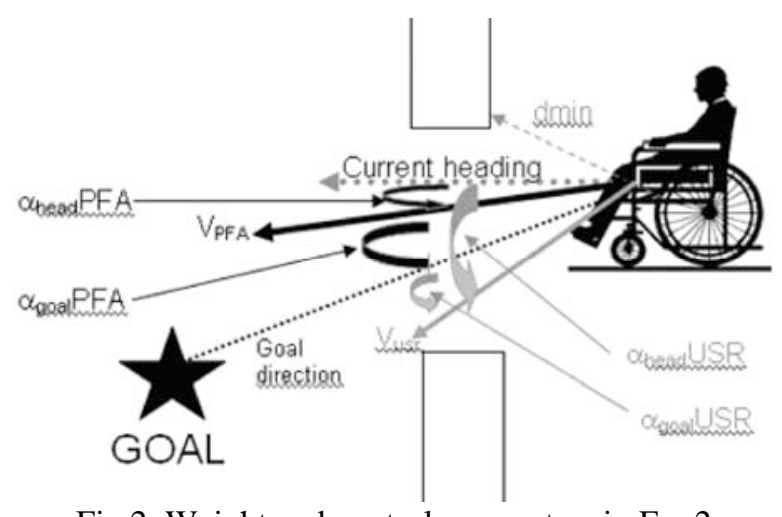

Fig.2. Weight and control parameters in Eq. 2

\section{EXPERIMENTS AND RESULTS}

The following experimentsfocused on checking the combined performance of human and robotic control in driving a wheelchair in Fondazione Santa Lucia ${ }^{1}$ (FSL) in Rome. Tests were performed using a commercial Meyra wheelchair donated by Sauer Medica that we previously equipped with a laser and wheel encoders and an onboard computer. The joystick of the chair had been replaced with another one that derived the commands to an onboard industrial PC before sending it to the motors. The PC modified the joystick commands to provide assistance. We call this chair CARMEN (Collaborative Autonomous Robot for Motion Enhancement and Navigation).

All tests were performed by volunteers that presented different degrees of physical and/or cognitive disabilities, previously evaluated by the doctors in FSL. A doctor and an engineer were present at all times and, for these experiments, the hall where the patients moved was isolated from non authorized people. PFA were adjusted to grant a minimum distance to obstacle approximately equal to $20 \mathrm{~cm}$ to allow door crossing. If obstacles were detected closer than this distance, an underlying safety layer stops the robot.

The goal of these experiments is to check if the system is capable of adapting assistance to the user's needs at each moment. As a second target, we wanted check if shared control improves the drive learning process. Hence, we measure the amount of help provided to the user in each trajectory, his/her stand-alone efficiency, the time required to finish the trajectory and asked about the subjective degree of satisfaction of each user.

\section{${ }^{1}$ http://www.hsantalucia.it/}

The presented experiments have been designed taking into account the conclusions provided by Yanco et al in HRI evaluations [12]. According to their results, the major shortcomings in this kind of experiments are that: i) system designers are often enlisted as test users; ii) HRI evaluations are commonly informal and rarely provide controlled, objective assessment; iii) there is a very limited number of users. We asked more than 30 patients presenting different conditions to perform the experiments and used well known metrics to evaluate results. These metrics are briefed in Table I. Metrics have been divided into two types: Task, refererred to physical aspects and Psycho, referred to cognitive aspects [13].

TABLE I

METRICS IN THE EXPERIMENT

\begin{tabular}{|c|c|c|}
\hline Metric & Type & Relates to \\
\hline Smoothness & Task & Sharp direction changes \\
Safety & Task & Closeness to obstacles \\
Directiveness & Task & Keeping the goal ahead \\
Curvature & Task & global trajectory smoothness \\
Time to destination & Task & Total time to complete the path \\
\hline Intervention level & Psycho & Collaboration with machine \\
Disagreement & Psycho & Effort and frustration \\
Consistency & Psycho & Cognitive capabilities \\
Joystick variation & Psycho & Workload \\
Questionnaire & Psycho & Subjective opinion \\
\hline
\end{tabular}

Performance, in absence of a canon trajectory clearly marked on the floor, can not be measured via error with respect to a given trajectory. Hence, in the current experiments we decided to simply characterize the efficiencies of human, machine and shared control at each point via local parameters, as specified in previous sections: safety, directiveness and smoothness. Thus, following a fixed path is not as important as driving well. We also use some global parameters to measure the emergent trajectory without a canon one. A simple parameter of this kind is the maximum/minimum of the aforementioned local parameters. We have also chosen Trajectory Curvature, a parameter that measures how much a curve bends at each point. Another common parameter is the well known Time to destination, which we use to evaluate if a given person reduces his/her own task time with practise

Psycho metrics in absence of biometrics sensors can only be indirectly measured. Intervention level is defined as the portion of time that the user moves a joystick. In collaborative control, a high intervention level is desired, meaning that the system is highly cooperative. We have chosen to evaluate person/wheelchair agreement via a Disagreement parameter, which represents the difference between the human output and the robot output. Since both outputs are vectors, we measure Disagreement in terms of angles. In our target population, Consistency is also important. It is defined as the variation of the user output when facing similar situations. A high Consistency is expected to be related to users with good cognitive capabilities, whereas a low one is related to random joystick motion. Another parameter related to user control is Joystick Variation, which measures a change of more than $10 \%$ in the position of the stick. This information has been used as an indirect measure of workload. It is important to note, though, that it may be also related to spasmodic joystick movements. 
Metrics for all the experiments performed are briefed in Table II. It can be observed that, in brief, Task metrics are fairly high using the proposed approach, even though they present significant variations from person to person. The highest one is clearly Safety, which is preserved via PFA, and the lowest is Directiveness, that largerly depends on cognitive and physical abilities of the users. Regarding psycho metrics, a high Intervention level is achieved. Disagreement is fairly low and we have checked that it mostly depends on the cognitive capabilities of the user: the better these capabilities, the larger the Disagreement. Inconsistency is also fairly low and mostly related to low cognitive capabilities. Finally, Joystick Variation is very low, meaning that the interface

TABLE II

METRIC RESULTS

\begin{tabular}{|c|c|c|c|}
\hline Metric & Type & Average & Deviation \\
\hline Smoothness & Task & 78.72 & 25.501 \\
Safety & Task & 95.48 & 16.13 \\
Directiveness & Task & 57.15 & 27.13 \\
Curvature & Task & -0.11 & 3.78 \\
\hline \hline Intervention level & Psycho & 84.07 & \\
Disagreement & Psycho & 25.13 & 19.94 \\
Inconsistency & Psycho & 13.37 & 13.94 \\
Joystick variation & Psycho & 2.19 & 7.31 \\
\hline
\end{tabular}

is not supposed to be stressful to the users.

Fig. 3 shows paths followed by the different users in the first experiment (move from corridor to a room). It can be observed that paths are initially very similar, but they change by the door area. This occurs because no turning point was specified: the users were only instructed to turn left and cross the door.

Fig. 4 presents a representation of the three local efficiencies used to calculate how much control person and robot have at each point, as well as the global one: each efficiency (directiveness, smoothness and safety) becomes the R, G and B channel of the RGB colour space. If all efficiencies are high, the colour of a given point of the trajectory is high and white. Pure colours correspond to two low factors and combined colours to a decrease in a single factor. It can be observed that reactive control is not good for door crossing (Fig. 4.a), as reported in pure PFA. The user does not perform so well (Fig. 4.b), especially when she needs to turn right to position herself in the corridor. Until the end of the trajectory, softness is not recovered. However, she was better than the robot to go through the door, as she was careful with respect to distance and moved in a straight way. In this case, the full potential of shared control can be observed in Fig. 4.c, as it clearly improves performance not only the user's but also the one of the robot and equalizes them.

As commented, coming in or out of the door is not a symmetric task. It is actually harder to come from the corridor, turn and cross the door than to come from the room to the corridor and then, turn. In the first case, the

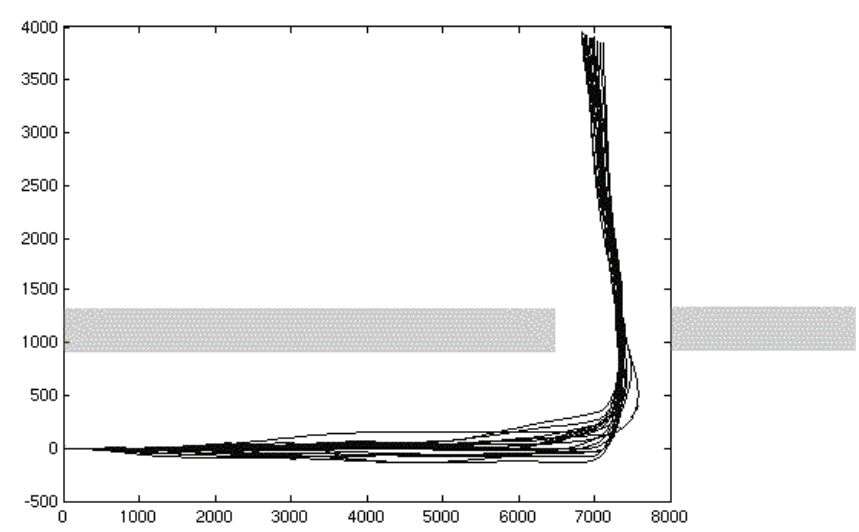

Fig. 3. Room entering trajectories

turning point is a key decision to successfully cross the door, whereas in the second one, it is just a matter of facing the corridor closer to the wall or to the center. This can be clearly appreciated if the performance of different persons are evaluated isolately. For example, we can check the performance of in-patient 28, with an MMSE [14] equal to 20, GDS [15] equal to 10 and Barthel [16] equal to 41 , roughly meaning that he has significant physical, yet not cognitive disabilities, mild geriatric depression and requires assistance for daily living.

This in-patient had more trouble, as expected, to turn before actually crossing the door, as reflected in the average time to complete the trajectories $(49,74$ and 62.87 seconds respectively). It was also to be expected that efficiency reduction in this case was related to a lower smoothness, as he performed sharper direction changes to face the door before crossing it to the room. It is interesting to note, though, that shared control keeps average efficiencies quite similar in in and out trajectories, even though psycho metrics are indeed significantly different. The in-patient presents in both cases a high intervention level, but disagreement almost duplicates to move into the room and presents a large variance. Nevertheless, the joystick variation for this in-patient was not that large, meaning that disagreement does not provoke frequent sharp joystick changes and, consequently, the in-patient is not expected to be too stressed.

In general, we appreciated that in-patients tended to quickly learn how to control the chair through use, even for harder tasks. This effect was far more evident in persons who found it hard to control the wheelchair due to lack of experience or mild functional disability. For example, in-patient 5 (MMSE=28, GDS=8, Barthel=69) went down from $110.56 \mathrm{~s}$ to exit the door the first time (10.8 meters) to 56.01 (10.60 meters). Door entering was, as commented, a little harder. In this case, the in-patient reduced the initial time of $195.90 \mathrm{~s}$. (24.66 meters) to $53.81 \mathrm{~s}$ (11.01 meters). The travelled distance reduction also indicates that this final run was far more efficient that the initial one. This was also evidenced by lower 


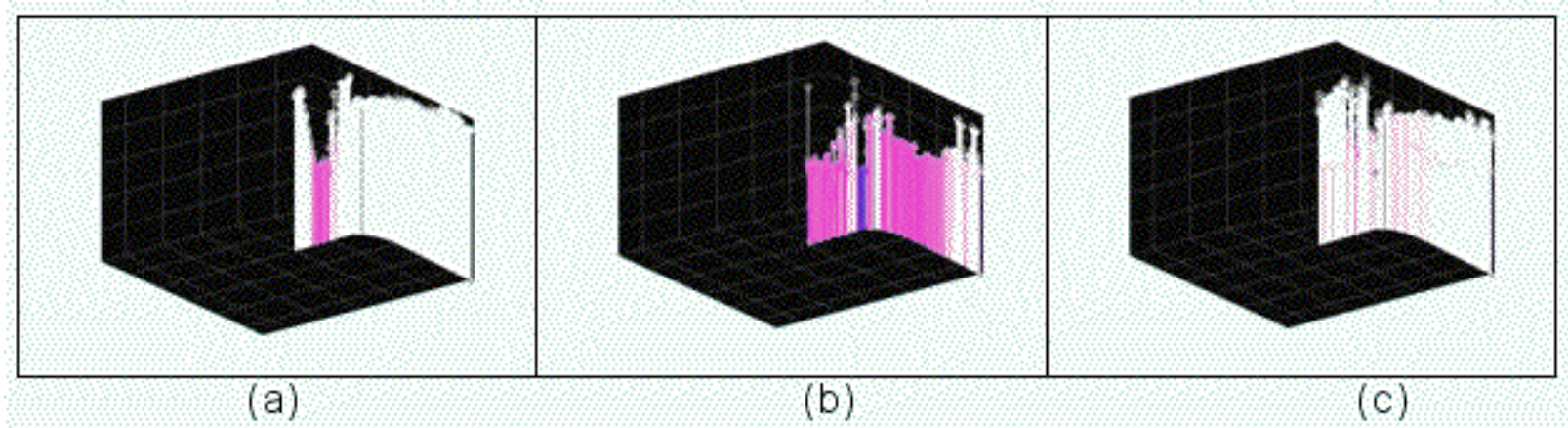

Fig. 4. Efficiencies to cross the door for: a) robot; b) human; c) collaborative control

Disagreement and Joystick variation in latest runs of the experiment for the same user. If we evaluate time evolution for all users in every test, it takes them 6.9 seconds less in average, with a variation of $21.1 \mathrm{~s}$, to reach their target. This average is not too meaningful, as time required for different trajectories is different as well, but it reflects the fact that experience makes users take less time to complete their tasks even from a rough point of view.

Similar effects could be observed in all patients, concluding that shared control helped them to control the wheelchair and allowed door crossing in all cases despite their condition. Qualitatively, all volunteers filled a questionnaire and acknowledged that they were satisfied with the wheelchair performance, even though in some cases they realized it was not completely obeying them.

\section{CONCLUSION}

This paper has presented a new approach to shared control for collaborative wheelchair navigation. The key idea of this work is to locally measure the efficiency of both human and autonomous chair navigation algorithm at each time instant. Using the two efficiencies, human and robot commands can be weighted and linearly combined into a single, emergent motory command.

Continuous combination of these commands returns a combined behavior that does not match exactly the robot or human stand alone performance but tends to combine them in a seamless way. We have tested the system with more than 30 volunteers presenting different disabilities ranging from minor to mild. Navigation is based on a purely reactive PFA, so that humans may also enhance the performance of the robot regarding oscillations and local minima. We wanted users to cooperate as much as possible to avoid loss of residual capabilities when they make no effort. In fact, their intervention level was above $90 \%$ in almost all cases. Furthermore, all users were able to complete the suggested tasks and their opinion on the wheelchair was very positive.

\section{ACKNOWLEDGMENT}

This work has been partially supported by the Spanish Ministerio de Educación y Ciencia (MEC) TEC200611689 and the European Union project SHARE-it (FP6045088). The authors would like to thank FSL and all volunteers for their kind cooperation and Sauer Medica for donation of the original wheelchair to build CARMEN.

\section{REFERENCES}

[1] Frese U, Larsson P, Duckett T, "A multigrid algorithm for simultaneous localization and mapping, IEEE Transactions on Robotics 21(2):1(125-64), 2005.

[2] Miller D, Assistive robotics: an overview, Springer-Verlag, pp 126136, 1998

[3] Mandel C, Huebner K, Vierhu® T (2005)" Towards an autonomous wheelchair: Cognitive aspects in service robotics", Proceedings of Towards Autonomous Robotic Systems (TAROS 05) pp 165-172, 2005

[4] Simpson R, Levine SP, NavChair: An Assistive Wheelchair Navigation System with Automatic Adaptation, Springer-Verlag, pp 235-255, 1998

[5] Horiguchi Y, Sawaragi T, "Effects of probing to adapt machine autonomy in shared control systems". In: Proc. International Conference on Systems, Man and Cybernetics, Hawaii, USA, vol 1, pp 317-323, 2005

[6] Bruemmer DJ, Few DA, Boring RL, Marble JL, Walton MC, Nielsen CW, "Shared understanding for collaborative control". IEEE Transactions on Systems, Man and Cybernetics - Part A: Systems and Humans, 25(4):494-504, 2005

[7] Gomi T, Griffith A, Developing Intelligent Wheelchairs for the Handicapped, Springer-Verlag, pp 150-178, 1998

[8] Simpson R, Levine S "Development and evaluation of voice control for a smart wheelchair". In: Proc. Annual RESNA Conf., Washington, USA, pp 417-419, 1997

[9] Crisman J, Cleary M, Progress on the deictic controlled wheelchair, Springer-Verlag, pp 137-149, 1998

[10] Bourhis G, Agostini Y ”The VAHM robotized wheelchair: System architecture and human-machine interaction" Journal of Intelligent Robotic Systems 22(1):39-50, 1998

[11] Khatib O, "Real-time obstacle avoidance for manipulators and mobile robots. International Journal of Robotics Research 5(1):9098, 1986

[12] Yanco HA, Drury JL, Scholtz J, "Beyond usability evaluation: Analysis of human robot interaction at a major robotics competition". Journal of Human Computer Interaction 19:117-149, 2004

[13] Cooperstock J, Pineau J, Precup D, Atrash A, Jaulmes R, Kaplow R, Lin N, Prahacs C, Villemure J, Yamani H, "Smartwheeler: A robotic wheelchair test-bed for investigating new models of humanrobot interaction". In: Proc. of the IEEE Conference on Intell. Robots and Systems (IROS), San Diego, USA, 2007

[14] Crum R, Anthony J, Bassett S, Folstein M, "Population-based norms for the mini-mental state examination by age and educational level". Journal of the American Medical Association 269(18):2386239, 1993

[15] Yesavage J, Brink T, Rose T, Lum O, Huang V, Adey M, Leirer V "Development and validation of a geriatric depression screening scale: A preliminary report. Journal of Psychiatric Research 17:3749, 1983

[16] Mahoney F, Barthel D "Functional evaluation: the Barthel index". Maryland State Medical Journal (14):56-61, 1965 